\title{
Finite-Size Effects in Tunneling between Parallel Quantum Wires
}

\section{Citation}

Tserkovnyak, Yaroslav, Bertrand I. Halperin, Ophir M. Auslaender, and Amir Yacoby. 2002. “Finite-Size Effects in Tunneling between Parallel Quantum Wires." Physical Review Letters 89 (13). https://doi.org/10.1103/physrevlett.89.136805.

\section{Permanent link}

http://nrs.harvard.edu/urn-3:HUL.InstRepos:41417280

\section{Terms of Use}

This article was downloaded from Harvard University's DASH repository, and is made available under the terms and conditions applicable to Other Posted Material, as set forth at http:// nrs.harvard.edu/urn-3:HUL.InstRepos:dash.current.terms-of-use\#LAA

\section{Share Your Story}

The Harvard community has made this article openly available.

Please share how this access benefits you. Submit a story.

Accessibility 


\title{
Finite-size effects in tunneling between parallel quantum wires
}

\author{
Yaroslav Tserkovnyak, ${ }^{1}$ Bertrand I. Halperin, ${ }^{1}$ Ophir M. Auslaender, ${ }^{2}$ and Amir Yacoby ${ }^{2}$ \\ ${ }^{1}$ Lyman Laboratory of Physics, Harvard University, Cambridge, Massachusetts 02138 \\ ${ }^{2}$ Dept. of Condensed Matter Physics, Weizmann Institute of Science, Rehovot 76100, Israel
}

(Dated: October 28, 2018)

\begin{abstract}
We present theoretical calculations and experimental measurements which reveal finite-size effects in the tunneling between two parallel quantum wires, fabricated at the cleaved edge of a GaAs/AlGaAs bilayer heterostructure. Observed oscillations in the differential conductance, as a function of bias voltage and applied magnetic field, provide direct information on the shape of the confining potential. Superimposed modulations indicate the existence of two distinct excitation velocities, as expected from spin-charge separation.
\end{abstract}

PACS numbers: 73.21.Hb,71.10.Pm,73.23.Ad,73.50.Jt

One-dimensional (1D) electronic systems are a very fertile ground for studying the physics of interacting many-body systems. In one dimension, the elementary excitations are collective spin and charge modes, the spectrum of which is strongly influenced by the Coulomb interaction [1]. An electron entering such a system must, therefore, decompose into the corresponding eigenmodes, resulting in a suppression of the tunneling density of states. This suppression was detected in a variety of experiments, such as tunneling from metal contacts into carbon nanotubes [2] and resonant tunneling in one dimension [3]. A unique feature of interacting electrons in one dimension, described by Luttinger-liquid (LL) theory [1], is the decoupling of the spin and charge degrees of freedom, each of which propagates with a different velocity determined by the Coulomb interaction. To date, direct experimental verification of this phenomenon is lacking. Moreover, issues pertaining to the decoherence and relaxation of the elementary excitations of the LL remain

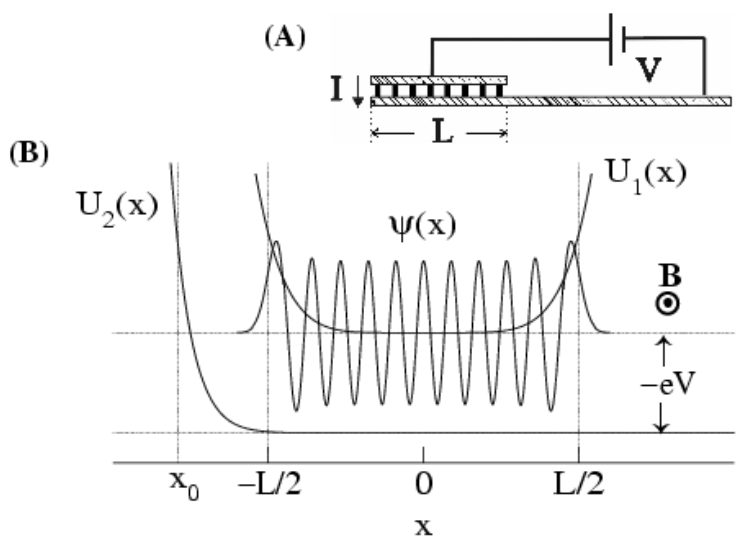

FIG. 1: Schematics of the circuit (A) and the model (B). A wire of length $L$ runs parallel to a semi-infinite wire. Boundaries of the wires are formed by potentials $U_{1}(x)$ and $U_{2}(x)$. Also drawn in (B) is a one-electron wave function $\psi(x)$ in the upper-wire confinement $U_{1}(x)$. The energy and momentum of the tunneling electrons are governed by voltage $V$ and magnetic field $B$. a challenge both theoretically and experimentally.

Here we report a detailed experimental investigation and theoretical explanation of a set of interference patterns in the nonlinear tunneling conductance between two parallel wires that were first reported in Ref. [4]. A sketch of the tunneling geometry in shown in Fig. 11(A). The interference appears because the tunneling process is coherent to a very high degree and is due to the finite length of the tunnel junction. A wealth of information can be extracted from the interference: The pattern itself encodes microscopic details of the potentials in the wires, while the structure of its envelope reflects the presence of two distinct excitation velocities per electron mode in the data, as expected from spin-charge separation. The decay of interference may also yield information on decoherence processes of the elementary excitations in $1 \mathrm{D}$ systems.

Fabrication of 1D quantum wires of exceptional quality has been achieved by cleaved edge overgrowth in GaAs/AlGaAs heterostructures [5], and much progress has been made in experimental investigations of their transport properties [4, 6]. Recently, a method was developed to measure tunneling between two parallel wires of this type [ $\llbracket$ : The wires are formed at the cleaved edge of a wafer containing two parallel quantum wells, only one of which is occupied by a two-dimensional (2D) electron gas (2DEG) (cf. Ref. 㳊 for details on the sample). A voltage bias $V$ between the wires forces electrons to tunnel through a narrow AlGaAs barrier separating them [see Fig. [1(A)]. Measurements of the differential conductance $G$ at $0.25 \mathrm{~K}$ are made with standard lock-in techniques, as a function of $V$ and $B$, a magnetic field applied perpendicular to the plane containing the wires. This allows to determine the complete dispersion relations of the elementary excitations in the quantum wires $[1$. Prominent features of the measured $G(V, B)$ can be understood in terms of a model which considers two infinite parallel wires, and accounts for the electron-electron interactions by means of LL theory [1, 4, 4]. On the other hand, an observed oscillation pattern results from the finite length of the upper wire (UW). We show that key features of the 
oscillations can be understood assuming that the ends of the UW are defined by a soft confining potential, rather than assuming sharp, square-well confinement. Most of our discussion will employ a model of noninteracting electrons, which explains the most prominent features of the interference patterns. Interaction effects will be discussed at the end. Fig. 11(B) schematically shows the potentials $U_{1}(x)$ and $U_{2}(x)$ felt by electrons in the wires. Electrons in the UW are confined to a region of finite length by top gates at both ends of the junction. Electrons in the lower wire (LW) are reflected at the left end, but can pass under the right-hand gate, rendering it semi-infinite. The effective tunneling region is determined by the length of the UW, which is approximately the region $|x|<L / 2$ in Fig. 1(B). The magnetic field gives a momentum boost $\hbar Q=e B d$ to electrons tunneling from the UW to the $\mathrm{LW},-e$ being the electron charge and $d-$ the distance between the centers of the wires; $V>0$ favors tunneling of electrons from the LW to the UW.

As observed in Ref. 偖, the differential conductance has strong peaks along a set of curves in the $V$ - $B$ plane, where tunneling can occur with conservation of energy and momentum between a Fermi point in one wire and an electron mode in the other wire. Here we focus on the region near the intersection of the dispersion peaks in $G(V, B)$ at zero magnetic field [4] and voltage, $V=\left(E_{\mathrm{F}_{2}}-E_{\mathrm{F}_{1}}\right) / e$, necessary to overcome the difference in Fermi energies $E_{\mathrm{F}_{1}}$ and $E_{\mathrm{F}_{2}}$ of the UW and $\mathrm{LW}$, respectively. (In the experiments, several electron modes may be occupied in the wires. Here we consider only the mode in each wire with the largest Fermi momentum along the wire.) The most spectacular manifestation of the breaking of translational invariance is the appearance of a regular pattern of oscillations away from the dispersion curves. Fig. 2 shows typical examples of the patterns measured at low magnetic field. The lines that correspond to the dispersions appear as pronounced peaks that extend diagonally across the figure. Additionally, we observe numerous secondary peaks running parallel to the dispersions. These side lobes are asymmetric: They always appear to the right of the principal dispersion peaks. The result is a checkerboard of oscillations in region I, stripes in region II, and no regular pattern in region III (see Fig. 2). When the lithographic length $L$ is increased from $2 \mu \mathrm{m}$ [cf. Fig. 2(B)] to $6 \mu \mathrm{m}$ [cf. Fig. 2[(C)], the frequency in $V$ and $B$ increases by a factor of $\approx 3$. The period is approximately related to $L, d$, and the Fermi velocity $v_{\mathrm{F}}$ by $|\Delta V| L / v_{\mathrm{F}}=|\Delta B| L d=2 \pi \hbar / e$. Upon close examination of Figs. 2(B) and 2(C), one can discern a modulation of the interference that causes a series of faint streaks parallel to the $B$-axis, where the oscillations are suppressed. The strength of this modulation is shown in Fig. 2(A). As explained below, this is a moire pattern created by two superimposed sets of interference, each resulting from a distinct velocity that is present in the data.

We base our theoretical analysis on a phenomenologi- cal tunneling Hamiltonian

$$
H_{\text {tun }}=T \sum_{s} \int d x \Psi_{s 1}^{\dagger}(x) \Psi_{s 2}(x) e^{-i Q x}+\text { H.c. },
$$

where $\Psi_{s i}(x)$ is the spin-s electron field operator for the $i$ th wire $(i=1$ denotes the UW and $i=2$ the LW). Since the Zeeman energy in GaAs is small, we ignore the spin degrees of freedom, and characterize the electrons in the UW by a discrete set of energy eigenstates $\psi_{n}(x)$. The eigenstates in the lower wire form a continuum, which we write as $\varphi_{k_{2}}(x)$, indexed by wave vector $k_{2}$. Treating tunneling to the lowest nonvanishing order in perturbation, we find for the current

$$
I \propto \operatorname{sgn}(V)|T|^{2} \sum_{m}|M(n, Q, V)|^{2},
$$

where $n=n_{\mathrm{F}}+\operatorname{sgn}(V) m$, with $n_{\mathrm{F}}$ being the index of the state $\psi_{n}$ just below $E_{\mathrm{F}_{1}} ; \sum_{m}$ is a sum over integers

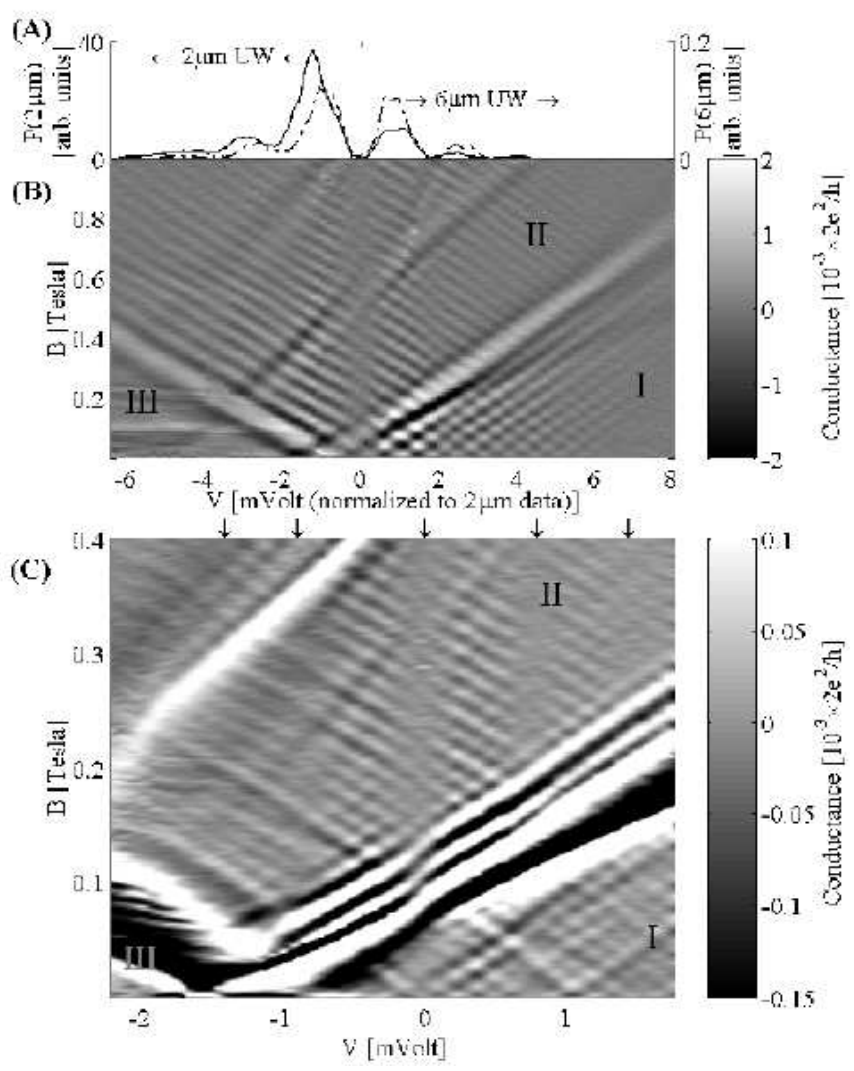

FIG. 2: Oscillations of $G(V, B)$ at low field from $2 \mu \mathrm{m}$ (B) and $6 \mu \mathrm{m}(\mathrm{C})$ junctions. (A smoothed background has been subtracted to emphasize the oscillations.) The arrows in (C) mark the minima of the power $P$ of the oscillations in magnetic field as a function of the voltage $V$. (A) shows $P$ for (B) (solid line) and (C) (dashed), for which the abscissa is $2.3 \times V$. The brightest lines in (B) and (C), corresponding to tunneling between the lowest modes, break the $V-B$ plain into regions I, II, and III. Additional bright lines in II arise from other 1D channels in the wires and are disregarded in our theoretical analysis. 
$m$ with $[\operatorname{sgn}(V)-1] / 2<m<e|V| L / \pi \hbar v_{\mathrm{F}}$; and $M$ is the tunneling matrix element between state $\psi_{n}$ and state $\varphi_{k_{2}}$, the energy of which is lower by $e V$. Specifically, $M$ is given by

$$
M=\int d x \psi_{n}^{*}(x) e^{-i Q x} \varphi_{k_{2}}(x) .
$$

If $e|V|$ is not too large, we can linearize about the Fermi wave vectors $k_{\mathrm{F}_{i}}$, and $k_{2}$ is then given by $\left(k_{2}-k_{\mathrm{F}_{2}}\right) v_{\mathrm{F}_{2}}=$ $\operatorname{sgn}(V) v_{\mathrm{F}_{1}} m \pi / L-e V / \hbar$.

As a starting point, we consider infinite square-well confinement in the region $|x|<L / 2$ of the UW, so that $\psi_{n} \propto \sin n \pi(x / L-1 / 2)$. We also assume that the potential in the LW is infinite for $x<x_{0}$, so that the states in the LW have the form $\varphi_{k_{2}} \propto \sin k_{2}\left(x-x_{0}\right)$. In line with the experiments, we assume that $k_{\mathrm{F}_{1,2}}$ differ slightly by $\Delta k_{\mathrm{F}}=k_{\mathrm{F}_{1}}-k_{\mathrm{F}_{2}}$, but neglect the difference in the $v_{\mathrm{F}}$ 's of

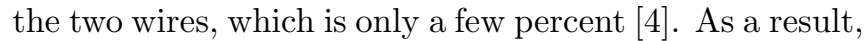
$|M|^{2}$ is independent of the index $n$ and is given by $|M|^{2}=$ $\left|M^{(+)}\right|^{2}+\left|M^{(-)}\right|^{2}$, where $M^{( \pm)}=\sin \left[\kappa^{( \pm)} L / 2\right] /\left[\kappa^{( \pm)} L / 2\right]$ and $\kappa^{( \pm)}=\Delta k_{\mathrm{F}}+e V / \hbar v_{\mathrm{F}} \pm Q$. (In the limit $L \rightarrow \infty$, the two terms $\left|M^{( \pm)}\right|^{2}$ become $\delta$-functions.) Similar results were obtained by Boese et al. [8] who considered tunneling between two infinite noninteracting wires through a window of finite length $L$ [9].

Differentiating Eq. (2) to obtain $G(V, B)$, we find that whenever the applied voltage matches a discrete energy level of the UW with the Fermi level of the LW, the sum in Eq. (22) exhibits a step, yielding a series of $\delta$-function peaks in the conductance. These peaks are not seen in the experiments, possibly because of smearing due to finite temperature and/or 1D-2D scattering in the UW. We may therefore disregard the discreteness of the sum and write $I \propto V|M(Q, V)|^{2}$, which gives a pronounced oscillatory contribution $\propto V \partial|M|^{2} / \partial V$ to $G$. (When $L \rightarrow \infty$, this oscillation pattern disappears and the conductance becomes $G \propto|M(Q, V)|^{2}$ resulting in bright peaks along the dispersion curves.) There are several features in the data, most notably the interference side-lobe asymmetry, not captured by this idealized model. The asymmetry of side lobes can be well understood if, instead of assuming square-well confinement, we consider a smooth potential well $U_{1}(x)$, giving rise to a WKB wave function form for the electronic states in the UW [see Fig. 1(B)].

As an example, we model the UW by a symmetric potential of the form

$$
U_{1}(x)=E_{\mathrm{F}_{1}}|2 x / L|^{\alpha} .
$$

The limit $\alpha \rightarrow \infty$ recovers the case of a square quantum well while a finite value of $\alpha \geq 2$ defines smooth walls. The appropriate choice of $\alpha$ should increase with $L$. As a specific example, we consider the case $\alpha=6$. We assume that the boundaries are soft enough so that one can use a WKB approximation for wave function $\psi_{n}$ in Eq. (3). We set $n_{\mathrm{F}}=100$ for the state at the Fermi energy in the UW,
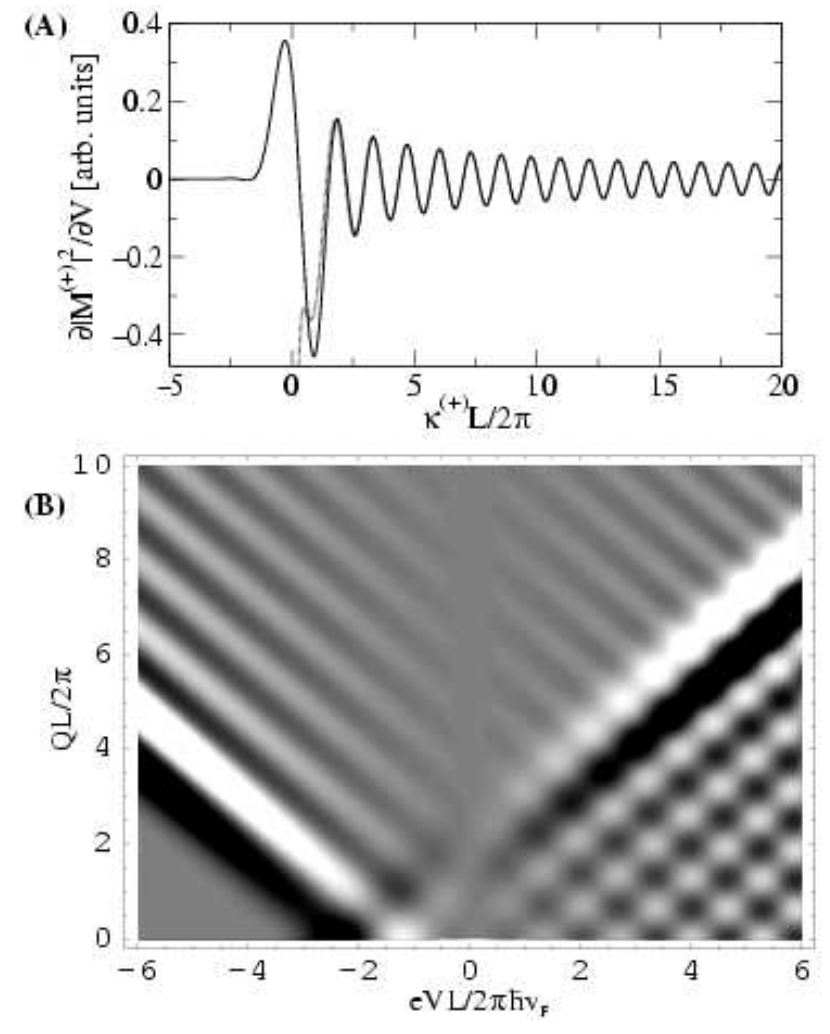

FIG. 3: (A) $\partial\left|M^{(+)}\right|^{2} / \partial V$, obtained numerically using the WKB wave function $\psi_{100}(x)$ and $\alpha=6$ in Eq. (4). The dotted curve shows an approximation [Eq. (B)]. (B) Calculated oscillation pattern $G \propto V \partial|M|^{2} / \partial V$ using the numerical result from Fig. B(A). $v_{\mathrm{F}_{1}}=v_{\mathrm{F}_{2}}=v_{\mathrm{F}}$ and $\Delta k_{\mathrm{F}}=4 \pi / L$.

which corresponds to electron density of $\approx 100 \mu \mathrm{m}^{-1}$ [4] in the most occupied 1D channel, with $L=2 \mu \mathrm{m}$. The theory again gives two separate contributions $\left|M^{( \pm)}\right|^{2}$ to $|M|^{2}$, which are functions of the variables $\kappa^{( \pm)}$, as before. In Fig. 3(A), we plot the results of a numerical calculation of $\partial\left|M^{(+)}\right|^{2} / \partial V$ as a function of $\kappa^{(+)}$. Unlike the square-well case, the smooth boundaries lead to a very asymmetric oscillation pattern for positive and negative $\kappa^{(+)}$. Furthermore, the amplitude of the oscillations in Fig. 3(A) drops much slower than in the case of infinitely steep walls. Results of a numerical calculation of $G(V, B)$ for the soft-confinement model, obtained from Eq. (3), are displayed in Fig. 3(B). They are very similar, at least qualitatively, to Fig. 2(B).

The numerical results can be understood analytically as follows. To find $M^{(+)}$, we substitute $e^{i k_{2} x}$ for $\varphi_{k_{2}}$ in Eq. (3), and replace $\psi_{n}$ by the right-moving WKB component $k(x)^{-1 / 2} e^{i k_{\mathrm{F}_{1}} x} e^{-i s(x)}$, where $k(x)=k_{\mathrm{F}_{1}}[1-$ $\left.U_{1}(x) / E_{\mathrm{F}_{1}}\right]^{1 / 2}$ and $s(x)=\int_{0}^{x} d x^{\prime}\left[k_{\mathrm{F}_{1}}-k\left(x^{\prime}\right)\right]$. Using $k_{2}-Q=k_{\mathrm{F}_{1}}-\kappa^{(+)}$, we see that if $0<\kappa^{(+)}<k_{\mathrm{F}_{1}}$, there are two points, $x^{+}>0$ and $x^{-}<0$, where $k(x)=k_{2}-Q$ and the integrand in Eq. (3) has a stationary phase. We evaluate the integral near these two points using the method of steepest descents, and add the results to ob- 
tain an estimate of $M^{(+)}$. In the case of a symmetric potential, $U_{1}(x)=U_{1}(-x)$, one finds

$$
M^{(+)} \approx \sqrt{\frac{16 \pi E_{\mathrm{F}} k_{\mathrm{F}}^{-2}}{U_{1}^{\prime}\left(x^{+}\right)}} \cos \left[\kappa^{(+)} x^{+}-s\left(x^{+}\right)-\frac{\pi}{4}\right] .
$$

In Fig. 3(A), we plot $\partial\left|M^{(+)}\right|^{2} / \partial V$, obtained using Eq. (5) as a dotted curve: it is in an excellent agreement with the full numerical calculation (solid line) for large positive $\kappa^{(+)}$. The stationary phase approximation (SPA) is bad for small values of $\kappa^{(+)}$, where the conditions for its validity are violated. For the potential of Eq. (化) $2 x^{+} / L \approx\left[2 \kappa^{(+)} / k_{\mathrm{F}}\right]^{1 / \alpha}$ for $\kappa^{(+)} \ll k_{\mathrm{F}}$, and the phase $\left[\kappa^{(+)} x^{+}-s\left(x^{+}\right)\right]$in Eq. (5) is equal to $\kappa^{(+)} x^{+} \alpha /(\alpha+1)$. For negative $\kappa^{(+)}$, the equation $k(x)=k_{2}-Q$ does not possess a real-valued solution, but after deforming the contour of integration [Eq. (3)] into the complex plane, we obtain a complex-valued solution $2 x / L \approx\left(2\left|\kappa^{(+)}\right| / k_{\mathrm{F}}\right)^{1 / \alpha} e^{-i \pi / \alpha}$. The argument of the cosine in Eq. (5) then has an imaginary part, which causes the magnitude of $\left|M^{(+)}\right|^{2}$ to rapidly fall by a factor of $\approx e^{-2 \pi^{2} / \alpha}$ over each period of oscillation. The approximation [Eq. (5)] can be used to predict both the period of the conductance oscillations and their amplitude. The period of the oscillations in the case of smooth confinement is given by $\Delta \kappa^{(+)} \approx 2 \pi /\left(x^{+}-x^{-}\right)$, rather than $\Delta \kappa^{(+)}=2 \pi / L$ for the square-well potential. In particular, this means that at low $\kappa^{(+)}$the period can be significantly larger than the value $2 \pi / L$, which it approaches for large $\kappa^{(+)}$. The prefactor $1 / U_{1}^{\prime}\left(x^{+}\right)$in $|M|^{2}$ falls off less rapidly with $\kappa^{(+)}$in the case of a smooth potential than the $1 /\left[\kappa^{(+)}\right]^{2}$ dependence for the square well. For Eq. (㓙), we find $1 / U_{1}^{\prime}\left(x^{+}\right) \propto\left[\kappa^{(+)}\right]^{1 / \alpha-1}$, which goes only as $1 / \kappa^{(+)}$for large $\alpha$ or $1 / \sqrt{\kappa^{(+)}}$for $\alpha=2$. (Because of the WKB approximation, we cannot recover the square-well result by setting $\alpha \rightarrow \infty$ in our expressions.) A larger period for small $\kappa^{(+)}$and relatively slow fall-off of the amplitude with increasing $\kappa^{(+)}$are both qualitatively consistent with the experimental results.

An analysis using the SPA and WKB approximations can also be applied to an interacting electron system in a pair of wires with soft confinement. The conductance is determined by Green's function $G_{12}=$ $\left\langle\left[\Psi_{s 2}^{\dagger} \Psi_{s 1}(x, t), \Psi_{s^{\prime} 1}^{\dagger} \Psi_{s^{\prime} 2}\left(x^{\prime}, 0\right)\right]\right\rangle$. The right- and leftmover contributions to $G_{12}$ can be approximated by the respective contributions $C_{R, L}^{\infty}$ for an infinite wire, given by LL theory [7], multiplied by phase factors of the form $e^{ \pm i\left[s(x)-s\left(x^{\prime}\right)\right]}$. For a pair of coupled, nearly identical wires, the leading singularities in $C_{R, L}^{\infty}$ occur at velocities $v_{c}$ and $v_{s}$, corresponding to charge and spin excitations that have opposite sign in the two wires, as the symmetric modes are not excited in the tunneling process.

We find that LL theory preserves the key qualitative features in Fig. 3(B), such as the asymmetry of the interference pattern, but brings about additional features resulting from the presence of two distinct velocities. In particular, the main dispersion peaks split into two lines with slopes defined by $v_{c}$ and $v_{s}$. As a consequence, the overlap of the interference side lobes formed along the dispersion slopes creates a moire pattern resulting in a periodic modulation of the conductance oscillations along the voltage axis. The distance between the corresponding stripes of suppressed conductance running parallel to the field axis is $\Delta V_{\text {slow }} \approx 2 \pi \hbar v_{c} v_{s} / e L\left(v_{c}-v_{s}\right)$, which is larger than the period of oscillations, $\Delta V_{\text {fast }} \approx$ $4 \pi \hbar v_{c} v_{s} / e L\left(v_{c}+v_{s}\right)$. Such a beating phenomenon in the interference is seen in Fig. 2 and may thus be a direct consequence of spin-charge separation in one dimension. For the $2 \mu \mathrm{m}$ wire, from the data in Figs. 2(A),(B), we estimate $\Delta V_{\text {slow }}=1.8 \pm 0.4 \mathrm{mV}$ and $\Delta V_{\text {fast }}=0.7 \pm 0.1 \mathrm{mV}$, and then find $v_{s} / v_{c}=0.67 \pm 0.07$. This result agrees with estimates made previously in a different regime [ $[$. A detailed comparison between theory and experiment can thus be used to study electron-electron interactions as well as the shape of the confining potentials along the quantum wires. Another effect predicted by LL theory, and present in the data, is a decrease in the value of $G$ at low bias, i.e., zero-bias anomaly. In the limit $L \rightarrow \infty$, the interference side lobes disappear, and what remains for the interacting wires are the split dispersion peaks, given by the LL theory of Ref. [7]. A more thorough discussion of LL theory for the double-wire system will be given elsewhere [10].

We have enjoyed illuminating discussions with B. Kramer, Y. Oreg, M. Sassetti, and A. Stern. This work was supported in part by NSF Grant DMR 99-81283, the Schlumberger Foundation, the US-Israel BSF, the Fusfeld Research Fund, and the Israeli Ministry of Science.

[1] J. T. Devreese, R. P. Evrard, and V. E. van Doren, eds., Highly Conducting One-Dimensional Solids (Plenum Press, New York, 1979); F. D. M. Haldane, J. Phys. C: Sol. State Phys. 14, 2585 (1981); J. Voit, Phys. Rev. B 47, 6740 (1993).

[2] M. Bockrath et al., Nature 397, 598 (1999).

[3] O. M. Auslaender et al., Phys. Rev. Lett. 84, 1764 (2000); H. W. C. Postma et al., Science 293, 76 (2001).

[4] O. M. Auslaender et al., Science 295, 825 (2002).

[5] A. Yacoby et al., Phys. Rev. Lett. 77, 4612 (1996); Solid State Commun. 101, 77 (1997); L. N. Pfeiffer et al., Microelectron. J. 28, 817 (1997).

[6] R. de Picciotto et al., Phys. Rev. Lett. 85, 1730 (2000); Nature 411, 51 (2001).

[7] D. Carpentier, C. Peça, and L. Balents, condmat/0103193 (unpublished); U. Zülicke and M. Governale, Phys. Rev. B 65, 205304 (2002).

[8] D. Boese et al., Phys. Rev. B 64, 085315 (2001).

[9] Tunneling through a window of finite length between a Luttinger wire and a 2DEG, but in a very different regime from that considered here, was discussed by M. Governale, M. Grifoni, and G. Schön, Phys. Rev. B 62, 15996 (2000). 
[10] Y. Tserkovnyak et al., in preparation. 